\title{
JORDAN DIVISION ALGEBRAS AND THE ALGEBRAS $A(\lambda)$
}

\author{
BY \\ CHARLES M. PRICE
}

1. Definition of Jordan algebras and the algebras $A(\lambda)$. Given any (not necessarily associative) algebra $A$ of finite dimension over a field $F$ and any $\lambda$ in $F$ we shall designate by $A(\lambda)$ the system consisting of the same vector space as $A$ but with a new multiplication o defined by $x \circ y=\lambda x y+(1-\lambda) y x$ for any $x, y$ in $A(\lambda)$, where $x y$ denotes the ordinary product in $A$. In particular, if the characteristic of $F$ is not two (which we shall assume throughout this paper), then $1 / 2$ is in $F$, and we shall use the special notation $A^{(+)}$for $A(1 / 2)$.

The center of $A$ is defined to be the set of all elements of $A$ which commute and associate with all elements of $A$. If $A$ has a unity element 1 , then $A$ is called central over $F$ provided its center is $1 F$.

A commutative algebra $B$ over $F$ is called a Jordan algebra provided $\left(x^{2} y\right) x=x^{2}(y x)$ for all $x, y$ in $B$. Then $B$ is said to be simple if the only nonzero ideal of $B$ is $B$ and $B$ is not the zero algebra of order one; $B$ is said to be a division algebra if $B$ contains no divisors of zero.

2. Central simple Jordan algebras. If $B$ is a simple Jordan algebra of finite dimension over $F$, then $B$ has a unity quantity 1 and its center is a field. It is known that $\left({ }^{1}\right)$ if $B$ is a central simple Jordan algebra of finite dimension over a nonmodular field $F$, then $B$ must be one of the following types:

Type 1. Let $A$ be a central simple associative algebra of finite dimension $m=r^{2}$ over its center $F$. Then $B=A^{(+)}$is a central simple Jordan algebra of order $m$ over $F$ and will be said to be of Type 1 .

Type 2. Let $A$ be as in Type 1 and suppose that $A$ has an involution $J$ of the first kind, that is, $J$ is an antiautomorphism of period two and $\alpha^{J}=\alpha$ for all $\alpha$ in $F$. Let $S$ be the set of all $J$ symmetric elements of $A$, that is, the set of all $a$ in $A$ such that $a^{J}=a$. Then $F \subset S$ and $B=S^{(+)}$is a central simple Jordan algebra (of Type 2) over $F$. The order of $S^{(+)}$over $F$ is known to be $r(r+1) / 2$ or $r(r-1) / 2$ depending on further properties of $J$, the latter possibility being excluded if $r$ is odd.

Type 3. Let $A$ be a central simple associative algebra of dimension $m=r^{2}$ over its center $K$. Let also $A$ have an involution $J$ of the second kind, that is, $J$ is an antiautomorphism of period two and $\alpha^{J} \neq \alpha$ for some $\alpha$ in $K$. Then the set of all $J$-symmetric elements of $K$ forms a subfield $F$ of $K$ such that

Presented to the Society, February 26, 1949, under the title Jordan division algebras; received by the editors February 20, 1950.

(1) See in this connection [3] and [4]. Numbers in brackets refer to the bibliography at the end of the paper. 
$[K: F]=2$. Designate by $S$ the set of all $J$-symmetric elements of $A$. Then $B=S^{(+)}$is a central simple Jordan algebra of dimension $m$ over $F$ and will be said to be of Type 3 .

Type 4. $B$ is an algebra of degree 2 over $F$ having a basis of the form $e$, $u_{1}, \cdots, u_{n-1}$ over $F$ such that $e u_{i}=u_{i} e=u_{i}, u_{i} u_{j}=u_{j} u_{i}=0, u_{i}^{2}=\alpha_{i} \neq 0, e^{2}=e$ for $i, j=1,2, \cdots, n-1 ; i \neq j, \alpha_{i}$ in $F, n \geqq 3$.

Type 5. Then $B$ is the so-called exceptional Jordan algebra $M$ of order 27 over its center, consisting of all three-rowed Hermitian matrices with elements in the Cayley numbers.

3. Plan of the paper. A Jordan division algebra may be trivially shown to be simple (see $[1$, p. 704]). Then any central Jordan division algebra of finite dimension over a nonmodular field must be one of the types listed above. Type 4 is obviously excluded. Also

$$
e_{11}=\left(\begin{array}{lll}
1 & 0 & 0 \\
0 & 0 & 0 \\
0 & 0 & 0
\end{array}\right) \quad \text { and } \quad e_{22}=\left(\begin{array}{ccc}
0 & 0 & 0 \\
0 & 1 & 0 \\
0 & 0 & 0
\end{array}\right)
$$

are in $M, e_{11} e_{22}=e_{22} e_{11}=e_{11} \circ e_{22}=0$ so that Type 5 is also excluded. The purpose of this paper is to attempt a derivation of the Jordan division algebras, in the sense that the simple Jordan algebras are derived (listed) above. In Part I we shall study the Jordan division algebras of Type 1 and the algebras $A(\lambda)$. Part II will deal with the Jordan division algebras of Types 2 and 3.

\section{Part I. Jordan division algebras of type 1 AND the Algebras $A(\lambda)$}

LemmA 1. Let $A$ be a simple associative algebra over $F$. Then a necessary condition that $A(\lambda)$ be a division algebra is that $A$ be a division algebra.

Since $A$ is simple, $A=M \times D$ where $M$ is a total matric algebra and $D$ is a division algebra. Suppose $M$ has degree $r>1$. Then $M$ has a basis $e_{i j}(i, j=1,2, \cdots, r), e_{i j} e_{k l}=\delta_{j k} e_{i l}$ where $\delta$ is the usual Kronecker function. Now $e_{11}, e_{22}$ are two nonzero elements of $A(\lambda), e_{11} e_{22}=e_{22} e_{11}=e_{11} \circ e_{22}=0, A(\lambda)$ is not a division algebra. Therefore if $A(\lambda)$ is a division algebra then $r=1$, $A=D, A$ is a division algebra.

Let $A$ be an associative algebra over $F$. For any $x$ in $A$ we shall designate by $A^{(x)}$ the set of all elements in $A$ which commute with $x$. Observe that $A^{(x)} \subset A^{\left(x^{2}\right)}$.

THEOREM 1. Let $A$ be an associative division algebra over $F$. Then $A^{(+)}$ is not a division algebra if and only if there exists an $x$ in $A$ such that $A^{(x)} \neq A^{\left(x^{2}\right)}$.

Suppose $A^{(+)}$is not a division algebra. Then there exists an $x \neq 0$ in $A^{(+)}$, $y \neq 0$ in $A^{(+)}$such that $x \circ y=(x y+y x) / 2=0, x y+y x=0, x y=-y x$. Also $x y \neq 0$, for $x y=0$ implies $x=0$ or $y=0$ from assumption that $A$ is a division algebra, contrary to choice of $x$ and $y$. If $x y=y x$ then from above $x y=y x$ 
$=-x y, 2 x y=0, x y=0$. This contradiction shows that $x y \neq y x, y \notin A^{(x)}$. However $x^{2} y=x(x y)=-(x y) x=y x^{2}, y \in A^{\left(x^{2}\right)}, A^{(x)} \neq A^{\left(x^{2}\right)}$. Conversely suppose that $A^{(x)} \neq A^{\left(x^{2}\right)}$ for some $x$ in $A$. Now since $A^{(x)} \subset A^{\left(x^{2}\right)}$, there exists a $z$ in $A^{\left(x^{2}\right)}$ such that $z$ is not in $A^{(x)}$. Then $z x^{2}=x^{2} z, u=x z-z x \neq 0, x \neq 0,2(x \circ u)$ $=x u+u x=x^{2} z-x z x+x z x-z x^{2}=0, A^{(+)}$is not a division algebra. This completes the proof of the theorem.

TheOREM 2. Let $A$ be a central associative division algebra of degree $n$ over $F$. Then if $A(\lambda)$ us not a division algebra, all of the following must hold:

(1) $\mu=-\lambda^{-1}(1-\lambda)$ is a primitive rth root of unity.

(2) $r$ divides $n$ so that $n=q r$ for some positive integer $q$.

(3) Any divisor of zero in $A(\lambda)$ has a minimum function of the form $\phi(\lambda)$, where $\phi(\lambda)$ is a polynomial in $\lambda^{r}$.

Suppose $A(\lambda)$ is not a division algebra. Then $a \circ b=\lambda a b+(1-\lambda) b a=0$ for some $a \neq 0, b \neq 0$ in $A$. If $\lambda=0$ then $b a=0$ for $a \neq 0, b \neq 0$ in $A$, contradicting the fact that $A$ is a division algebra. Similarly if $\lambda=1$ then $a b=0$ which leads to the same contradiction $\left({ }^{2}\right)$. Therefore we may assume that $\lambda \neq 0,1$. Now $a b=\mu b a$ where $\mu=-\lambda^{-1}(1-\lambda) \neq 0, a=\mu b a b^{-1}$ and by a trivial induction $a^{t}=\mu^{t} b a^{t} b^{-1}(t=0,1,2, \cdots)$. Let the minimum function of $a$ over $F$ be $\phi(\lambda)=\lambda^{m}+\alpha_{1} \lambda^{m-1}+\cdots+\alpha_{m}$ where the $\alpha_{i}$ are in $F(i=1, \cdots, m)$ and $\alpha_{m} \neq 0$. Then

$$
\begin{aligned}
\phi(a) & =a^{m}+\alpha_{1} a^{m-1}+\cdots+\alpha_{m}=0, \\
\phi\left(\mu b a b^{-1}\right) & =\phi(a)=\mu^{m} b a^{m} b^{-1}+\alpha_{1} \mu^{m-1} b a^{m-1} b^{-1}+\cdots+\alpha_{m}=0, \\
b^{-1} \phi\left(\mu b a b^{-1}\right) b & =\mu^{m} a^{m}+\alpha_{1} \mu^{m-1} a^{m-1}+\cdots+\alpha_{m}=0, \\
\mu^{m} \phi(a) & =\mu^{m} a^{m}+\alpha_{1} \mu^{m} a^{m-1}+\cdots+\mu^{m} \alpha_{m}=0 .
\end{aligned}
$$

Subtracting the latter from its preceding equality we get

$$
\alpha_{1}\left(\mu^{m-1}-\mu^{m}\right) a^{m-1}+\alpha_{2}\left(\mu^{m-2}-\mu^{m}\right) a^{m-2}+\cdots+\alpha_{m}\left(1-\mu^{m}\right)=0 .
$$

Since $\phi(\lambda)$ is the polynomial of least degree satisfied by $a$ we must have $\left(\mu^{m-i}-\mu^{m}\right) \alpha_{i}=0(i=1, \cdots, m)$. In particular, since $\alpha_{m} \neq 0,1-\mu^{m}=0, \mu^{m}=1$. Then for some $r$ dividing $m$ we have $\mu$ a primitive $r$ th root of unity. This proves (1) above. Also since $a$ generates a subfield of degree $m$ over $F, m$ must divide $n$. Therefore $r$ divides $n$ and we have proved (2). $\mu^{m-i}-\mu^{m}=\mu^{m-i}$ $-1=0$ if and only if $\mu^{i}=1$ which is true if and only if $i$ is a multiple of $r$. Therefore $\alpha_{i}=0$ for $i$ not a multiple of $r(i=1, \cdots, m), \phi(\lambda)$ is a polynomial in $\lambda^{r}$, which proves (3). q.e.d.

Note that for $\lambda=1 / 2, \mu=-2(1-1 / 2)=-1$ is a primitive square root of

(2) Note that if $\lambda=0, A(\lambda)$ is antiisomorphic to $A$; if $\lambda=1, A(\lambda)$ is isomorphic to $A$. For this reason we shall exclude the cases $\lambda=0,1$ from further consideration. 
unity and Theorem 2 then states that if $A^{(+)}$is not a division algebra, then $n$ must be even. We have proved the following corollary.

Corollary. Let $A$ be an associative central division algebra of odd degree over its center $F$. Then $A^{(+)}$is a Jordan division algebra.

Let $Q$ be a central simple associative algebra of degree two over its center $K$. Then it is well known that $Q$ is a so-called quaternion algebra, having a basis of the form $1, i, j, k=i j$ over $K$, with 1 acting as the identity (unity) quantity of $Q$ and multiplication defined by $i^{2}=\alpha$ in $K, j^{2}=\beta$ in $K, j i=-i j$; the remaining products being computed by means of the associative and distributive laws using these rules. Our only observation on $Q$ at this point shall be that $Q^{(+)}$contains divisors of zero; for example $i \circ j=(i j+j i) / 2=0$. We shall use this remark in the proof of the following theorem.

THEOREM 3. Let $A$ be an associative central simple algebra of degree $n$ over $F$ which is a crossed product, so that $A$ has a maximal subfield $N$ which is normal of degree $n$ over $F$. Then $A^{(+)}$is a division algebra if and only if $A$ is a division algebra and $n$ is odd.

Suppose $A^{(+)}$is a division algebra. Then by Lemma $1, A$ is a division algebra. Suppose $n=2 m$ is even. Then by Galois theory there exists a field $\bar{N}, F \subset \bar{N} \subset N$ such that $[N: \bar{N}]=2,[\bar{N}: F]=m$. Then the $A$ commutator of $\bar{N}$ (the set of all elements of $A$ which commute with every element in $\bar{N}$ ) is central simple of degree two over $\bar{N}$, and therefore is a quaternion algebra $Q$ which is contained in $A, Q^{(+)}$contains divisors of zero by the remark preceding the theorem which are contained in $A^{(+)}, A^{(+)}$is not a division algebra contrary to assumption above. This contradiction shows that $n$ must be odd.

Conversely suppose that $A$ is a central division algebra and $n$ is odd. Then $A^{(+)}$is a division algebra by the corollary to Theorem 2 .

THEOREM 4. Let $A$ be an associative central simple algebra of degree $n$ over $F$ which is cyclic, so that $A$ has a maximal subfield $Z$ which is cyclic of degree $n$ over $F$. Then $A(\lambda)$ is not a division algebra if and only if $A$ is not a division algebra or $\mu^{n}=1\left(\mu=-\lambda^{-1}(1-\lambda)\right)$.

Since $A$ is a cyclic, $A=Z+x Z+\cdots+x^{n-1} Z$ where $x^{n}=\gamma$ in $F, z x=x z^{S}$ for any $z$ in $Z$, where $S$ is the generating automorphism of the cyclic Galois group $G$ of $Z$ over $F$, that is, $G=\left[S, S^{2}, \cdots, S^{n}=I\right]$.

Now suppose $\mu^{n}=1$. Then $\mu$ is a primitive $r$ th root of unity and $n=q r$ for some positive integer $q$. Now $N_{Z: F}(\mu)=\mu^{n}=1$ and so by the Hilbert norm theorem there exists a $y \neq 0$ in $Z$ such that $y=\mu y^{S}, y^{S}=\mu^{-1} y$. Then $x \circ y$ $=\lambda x y+(1-\lambda) y x=\lambda x y+(1-\lambda) x y^{S}=\lambda x y+(1-\lambda) \mu^{-1} x y=\left[\lambda+(1-\lambda) \mu^{-1}\right] x y=$ 0 since $\lambda+(1-\lambda) \mu^{-1}=\lambda+(1-\lambda)(-\lambda)(1-\lambda)^{-1}=\lambda+(-\lambda)=0$. Since $x \neq 0$, $y \neq 0$ we have shown that $\mu^{n}=1$ implies $A(\lambda)$ is not a division algebra. If $A(\lambda)$ is a division algebra, then $A$ is a division algebra by Lemma 1 . This 
proves one half of Theorem 4. The other half is an immediate consequence of Theorem 2 .

It is known that associative central simple algebras of finite degree over an algebraic number field are cyclic. This yields directly the corollary:

CoROLlaRY 1. Let $A$ be an associative central simple algebra of degree $n$ over $F,[F: R]<\infty$ where $R$ is the field of all rational numbers. Then the conclusion of Theorem 4 holds.

Observe that in case $\lambda=1 / 2, \mu=-1, \mu$ is a primitive square root of unity, and the statement of Corollary 1 would read as in the following corollary.

Corollary 2. Let $A$ be as in Corollary 1. Then $A^{(+)}$is not a division algebra if and only if $A$ is not a division algebra or $n$ is even.

THEOREM 5. Let $A$ be an associative central simple algebra of degree $n(>1)$ over its center $F$, and suppose that $A$ has an involution $J$ of the first kind over $F$. Then $A^{(+)}$is not a division algebra.

We may assume that $A$ is a division algebra by Lemma 1. Suppose that $a=a^{J}$ for all $a$ in $A$. Then $(x y)^{J}=x y=y^{J} x^{J}=y x$ for all $x, y$ in $A, A$ is commutative, $A$ is a field, contrary to hypothesis $(n>1)$. Thus for some $b \in A$ we have $c=b-b^{J} \neq 0, c^{J}=b^{J}-b=-c, \quad\left(c^{2}\right)^{J}=\left(c^{J}\right)^{2}=c^{2}$. Then $F\left(c^{2}\right) \neq F(c)$. Let $\left[F\left(c^{2}\right): F\right]=t$. Then $[F(c): F]=2 t, n=2 t s$. Now $A^{(c)}$ is central simple of degree $s$ over $F(c)$ and so has order $2 s^{2} t$ over $F ; A^{\left(c^{2}\right)}$ is central simple of degree $2 s$ over $F\left(c^{2}\right)$ and so has order $4 s^{2} t$ over $F$. Since $4 s^{2} t \neq 2 s^{2} t$ we have $A^{(c)}$ $\neq A^{\left(c^{2}\right)}, A^{(+)}$is not a division algebra by Theorem 1 .

\section{PART II. JoRdAN DIVISION Algebras OF TYPES 2, 3}

We shall now prove a theorem which will serve us in the same manner as Lemma 1 of Part I, namely by reducing our study to the algebras $S^{(+)}$where $A$ is an associative division algebra, and $S$ is the set of all $J$-symmetric elements of $A$.

THEOREM 1. Let $A$ be an associative simple algebra of finite dimension over $F$ so that $A=M_{r} \times D$, where $M_{r}$ is a total matric algebra of degree $r$ over $F$, and $D$ is a division algebra. Let $J$ be an involution over $F$ of $A, S$ be the set of all $J$-symmetric elements of $A$. Then a necessary condition that $S^{(+)}$be a Jordan division algebra is that

(1) $r=1$ if $J$ is of the second kind,

(2) $r=1$ or 2 if $J$ is of the first kind.

In the latter case $S^{(+)}=D$ is a field.

Let $e_{i j}(i, j=1, \cdots, r)$ be the usual basis for $M_{r}$ over $F$, so that $e_{i j} e_{k l}$ $=\delta_{j k} e_{i l}$. Let $u=e_{i j}+e_{i j}^{J}$ for some fixed $i$ and $j$ with $i \neq j$. Then $u=u^{J}$ so that $u$ is in $S$. Suppose $u \neq 0$. Let $a=e_{i j} e_{i j}^{J}-e_{i j}^{J} e_{i j}, b=e_{i j} e_{i j}^{J}$. Since $a^{J}=a, b^{J}=b$, both $a$ and $b$ are in $S$. 
Case 1. $a=0$. Then $e_{i j} e_{i j}^{J}=e_{i j}^{J} e_{i j}$. If $b=0$ then $u \circ u=u^{2}=e_{i j}^{2}+e_{i j} e_{i j}^{J}+e_{i j}^{J} e_{i j}$ $+\left(e_{i j}^{J}\right)^{2}=e_{i j}^{2}+\left(e_{i j}^{2}\right)^{J}=0$ and $S^{(+)}$is not a division algebra, contrary to hypothesis.

Therefore we may assume $b \neq 0$. Then $b \circ b=b^{2}=\left(e_{i j} e_{i j}^{J}\right)^{2}=e_{i j}^{2}\left(e_{i j}^{J}\right)^{2}=0$ where we have used the fact that the hypothesis $a=0$ means that $e_{i j}$ and $e_{i j}^{J}$ commute with each other. But $b$ is in $S, b \neq 0, b \circ b=0, S^{(+)}$is not a division algebra, contrary to hypothesis. This contradiction shows that Case 1 cannot hold and we must have the following case.

Case 2. $a \neq 0$. Then $a \neq 0$ is in $S, u \neq 0$ is in $S, 2(a \circ u)=a u+u a=\left(e_{i j} e_{i j}^{J}\right.$ $\left.-e_{i j}^{J} e_{i j}\right)\left(e_{i j}+e_{i j}^{J}\right)+\left(e_{i j}+e_{i j}^{J}\right)\left(e_{i j} e_{i j}^{J}-e_{i j}^{J} e_{i j}\right)=e_{i j} e_{i j}^{J} e_{i j}+e_{i j}\left(e_{i j}^{J}\right)^{2}-e_{i j}^{J} e_{i j}^{2}-e_{i j}^{J} e_{i j} e_{i j}^{J}+e_{i j}^{2} e_{i j}^{J}$ $-e_{i j} e_{i j}^{J} e_{i j}+\left(e_{i j}^{J}\right)\left(e_{i j}\right)\left(e_{i j}^{J}\right)-\left(e_{i j}^{J}\right)^{2} e_{i j}=0$ since $e_{i j}^{2}=0=\left(e_{i j}^{2}\right)^{J}=\left(e_{i j}^{J}\right)^{2}$. Then $S^{(+)}$ is not a division algebra and Case 2 cannot hold. This contradicts our assumption that $u \neq 0$. Therefore we may assume that $u=0, e_{i j}=-e_{i j}^{J}$ for $i \neq j$. Suppose that $r>2$ so that we may find three distinct indices $i, j, k$. Then $e_{i j} e_{j k}=e_{i k}=\left(-e_{i j}^{J}\right)\left(-e_{j k}^{J}\right)=e_{i j}^{J} e_{j k}^{J}=\left(e_{j k} e_{i j}\right)^{J}=0$ which is impossible. Therefore $r \leqq 2, r=1$ or 2 . If $r=1$ then $A=D$ and we shall not discuss this case further here.

Now suppose that $r=2$ and $J$ is of the second kind. Then $\alpha \neq \alpha^{J}$ for some element $\alpha$ of the center of $A$. Then $\alpha^{J}$ is trivially in the center of $A$ so that $\theta=\alpha-\alpha^{J}=-\theta^{J} \neq 0$ is in the center of $A$. Also $\left(\theta e_{12}\right)^{J}=e_{12}^{J} \theta^{J}=\theta e_{12}$ since $\theta^{J}=-\theta$, $e_{12}^{J}=-e_{12}$. Therefore $\theta e_{12}$ is in $S, \theta e_{12} \neq 0,\left(\theta e_{12}\right) \circ\left(\theta e_{12}\right)=\left(\theta e_{12}\right)^{2}=\theta^{2} e_{12}^{2}=0, S^{(+)}$ is not a division algebra. Therefore if $J$ is of the second kind, $r=1, A=D$ and we have proved the first part of the theorem.

Suppose $r=2$ and $J$ is of the first kind. Now $e_{i j}^{J}=-e_{i j}(i, j=1,2)(i \neq j)$. Then $e_{i i}=e_{i j} e_{j i}=\left(-e_{i j}^{J}\right)\left(-e_{j i}^{J}\right)=e_{i j}^{J} e_{j i}^{J}=\left(e_{j i} e_{i j}\right)^{J}=e_{j j}^{J}$, that is, $e_{11}^{J}=e_{22}, e_{22}^{J}=e_{11}$. Therefore $M_{2}^{J}=M_{2}$ in the set theoretic sense, and since $D=A^{M_{2}}, D^{J} \subset D$; for if $d$ is in $D$ then $d$ commutes with all $m^{J}$ for $m$ in $M, d m^{J}=m^{J} d, m d^{J}=d^{J} m, d^{J}$ is in $A^{M_{2}}=D, D^{J} \subset D, D^{J}=D$ in the set theoretic sense. Suppose there is a $d$ in $D$ such that $d \neq d^{J}$. Then $c=d-d^{J} \neq 0$ is in $D, c^{J}=-c,\left(c e_{12}\right)^{J}=c^{J} e_{12}^{J}$ $=(-c)\left(-e_{12}\right)=c e_{12} \neq 0, c e_{12}$ is in $S,\left(c e_{12}\right)^{2}=c^{2} e_{12}^{2}=0=\left(c e_{12}\right) \circ\left(c e_{12}\right), S^{(+)}$is not a division algebra, a contradiction. We may thus assume that $d=d^{J}$ for every $d$ in $D$. Let $d_{1}, d_{2}$ be in $D$. Then $\left(d_{1} d_{2}\right)^{J}=d_{1} d_{2}=d_{2}^{J} d_{1}^{J}=d_{2} d_{1}, D$ is commutative, $D$ is a field. Let $a$ be in $A$. Then $a=d_{11} e_{11}+d_{12} e_{12}+d_{21} e_{21}+d_{22} e_{22}$ for $d_{i j}=d_{i j}^{J}$ in $D(i, j=1,2)$. Then $a^{J}=d_{11} e_{22}+d_{22} e_{11}-d_{12} e_{12}-d_{21} e_{21}$, so that $a=a^{J}$ if and only if $d_{12}=d_{21}=0$ and $d_{11}=d_{22}$ which is true if and only if $a=d_{11}\left(e_{11}+e_{22}\right)=d_{11}$ is in $D$, where we have used the fact that $e_{11}+e_{22}$ is the unity element of $D$, as well as that of $M_{2}$ and $A=M_{2} \times D$. Thus $S=D=S^{(+)}$is a field. This completes the proof of the theorem.

We shall now undertake the study of the case when $J$ is of the first kind and when $J$ is of the second kind in that order.

Lemмa 1. Let $A$ be an associative central division algebra of finite dimension over $F$ having an involution $J$ of the first kind. Suppose there exists an $x$ in $S$ such that $x^{2}$ is in $F, x$ is not in $F$. Then $S^{(+)}$is not a division algebra. 
Suppose that $S^{(+)}$is a division algebra.

Let $y$ be in $A$ and $y=-y^{J}$. Let $u=x y-y x$. Then $u^{J}=y^{J} x^{J}-x^{J} y^{J}=-y x$ $+x y=u$ so that $u$ is in $S$. Now $2(x \circ u)=x u+u x=x^{2} y-x y x+x y x-y x^{2}=0$ since $x^{2}$ is in $F, x \circ u=0$ for $x \neq 0$ and $u$ in $S$. Our supposition that $S^{(+)}$is a division algebra then implies that $u=0, x y=y x$. Thus $y=-y^{J}$ implies that $x y=y x$.

Let $z$ be in $A$ and $z=z^{J}$. Let $w=x z-z x$. Then $w^{J}=z x-x z=-w$ so that by the above italicized statement, we have $x w=w x, x^{2} z-x z x=x z x-z x^{2}$, $2 x^{2} z=2 x z x, 2 x(x z-z x)=0, x z=z x$ since $A$ is a division algebra and $x \neq 0$. We have shown $z=z^{J}$ implies $x z=z x$.

Any $a$ in $A$ may be written in the form $a=y+z$, where $z=\left(a+a^{J}\right) / 2$ $=z^{J}$ and $y=\left(a-a^{J}\right) / 2=-y^{J}$. Since $x$ commutes with both $z$ and $y, x$ commutes with $a, x$ is in the center $F$ of $A$ contrary to hypothesis. This contradiction shows that our supposition that $S^{(+)}$is a division algebra was incorrect, and so $S^{(+)}$is not a division algebra. q.e.d.

Lemma 2. Let $Q$ be an associative central division algebra of degree two over its center $F$ having an involution $J$ of the first kind. Then $S^{(+)}=F$ or $S^{(+)}$is not a division algebra.

By the remark preceding Theorem 3 of Part I we may assume that $Q$ has a basis $1, x, y, x y$ over $F$ with $x^{2}=\alpha$ in $F, y^{2}=\beta$ in $F, x y=-y x$. Now let $a$ be in $Q$ with $a^{2}=\delta$ in $F$. Then $a^{2}=\left(a^{2}\right)^{J}=\left(a^{J}\right)^{2}=\delta$. Let $u=a a^{J}-a^{J} a=u^{J}$ so that $u$ is in $S$. Then $a u+u a=a^{2} a^{J}-a a^{J} a+a a^{J} a-a^{J} a^{2}=0$ since $a^{2}=\delta$ in $F$. Applying $J$ to $a u+u a=0$ we get $u a^{J}+a^{J} u=0$. Adding the two we obtain $u\left(a+a^{J}\right)$ $+\left(a+a^{J}\right) u=2\left(a+a^{J}\right) \circ u=0$ for $a+a^{J}$ in $S$ and $u$ in $S$. Therefore $S^{(+)}$is not a division algebra unless either $a+a^{J}=0$ or $a a^{J}=a^{J} a$.

We now assume for the remainder of this proof that $S^{(+)}$is a division algebra. Then the preceding italicized statement implies that either $a+a^{J}=0$ or $a a^{J}=a^{J} a$. In either case $a a^{J}=a^{J} a, 0=a^{2}-\left(a^{J}\right)^{2}=\left(a-a^{J}\right)\left(a+a^{J}\right)$. Therefore $a= \pm a^{J}$ since $Q$ is a division algebra. We have proved that $a^{2}=\delta$ in $F$ implies that $a= \pm a^{J}$. In particular $x^{2}=\alpha$ in $F, y^{2}=\beta$ in $F$, and we have $x= \pm x^{J}$, $y= \pm y^{J}$.

Case 1. $x=x^{J}, y=y^{J}$. Then $x$ and $y$ are in $S, 2(x \circ y)=x y+y x=0$, contrary to our assumption that $S^{(+)}$is a division algebra. Therefore this case cannot occur.

Case 2. $x=x^{J}, y=-y^{J}$. Then $(x y)^{J}=y^{J} x^{J}=-y x=x y$ so that $x$ is in $S$, $x y$ is in $S, 2(x \circ x y)=x^{2} y+x y x=x(x y+y x)=0, S^{(+)}$is not a division algebra. Therefore this case cannot occur.

Case 3. $x=-x^{J}, y=y^{J}$. By symmetry with Case 2 (interchange $x$ and $y$ ), Case 3 cannot occur.

Case 4. $x=-x^{J}, y=-y^{J}$. Then $(x y)^{J}=y x=-x y$. Let $q=\gamma_{0}+\gamma_{1} x+\gamma_{2} y$ $+\gamma_{3} x y$ be any element of $S$. Then $q=q^{J}=\gamma_{0}-\gamma_{1} x-\gamma_{2} y-\gamma_{3} x y, \gamma_{1}=\gamma_{2}=\gamma_{3}=0$, $q=\gamma_{0}$ in $F, S=F=S^{(+)}$is a field. 
We have proved that if $S^{(+)}$is a division algebra, then $S^{(+)}=F$. This is merely a restatement of the theorem, and completes its proof. Note that the only case in which $S^{(+)}$is a division algebra, the involution $J$ is that which sends any $q$ in $Q$ into its conjugate.

THEOREM 2. Let $A$ be an associative central division algebra of finite degree $n>1$ over its center $F$ and let $A$ have an involution $J$ of the first kind so that $n=2^{m}$. Also assume that $A$ contains an imprimitive quartic field $K$ over $F$ when $n>2$. Then $S^{(+)}$is not a Jordan division algebra or $S^{(+)}$a field.

The case $m=1$ of this theorem follows from Lemma 2 . Hence assume $m>1$ and $S^{(+)}$is a division algebra. The hypothesis that $K$ is imprimitive means that there exists a subfield $L$ of $K$ such that $[K: L]=2,[L: F]=2$. Then, since the characteristic of $F$ is not two, we may take $L=F(r), K=L(s)$ where $r^{2}=\alpha$ in $F, \alpha$ not a square in $F$; $s$ primitive, $s^{2}=\beta+\gamma r$ in $L, \beta+\gamma r$ not a square in $L, \beta$ and $\gamma$ in $F, \gamma \neq 0$.

Let $u=r r^{J}-r^{J} r=u^{J}$ so that $u$ is in $S$. Then $2 u \circ\left(r+r^{J}\right)=\left(r+r^{J}\right) u$ $+u\left(r+r^{J}\right)=r^{2} r^{J}-r r^{J} r+r^{J} r r^{J}-\left(r^{J}\right)^{2} r+r r^{J} r-r^{J} r^{2}+r\left(r^{J}\right)^{2}-r^{J} r r^{J}=0$ where we have used the fact that $r^{2}=\left(r^{2}\right)^{J}=\left(r^{J}\right)^{2}=\alpha$ in $F$. The assumption that $S^{(+)}$is a division algebra then implies that either $u=0$ or $r+r^{J}=0$. In either case $r r^{J}=r^{J} r, 0=r^{2}-\left(r^{J}\right)^{2}=\left(r-r^{J}\right)\left(r+r^{J}\right)$ so that $r= \pm r^{J}$ since $A$ is a division algebra. If $r=r^{J}$, then $r^{2}$ is in $F, r$ is in $S, r$ is not in $F, S^{(+)}$is not a division algebra by Lemma 1 of Part II contrary to assumption. Thus $r=-r^{J}$, which means, incidentally, that $J$ is the ordinary conjugation operation in $L$. Now $s^{2}=\beta+\gamma r$ so that $\left(s^{2}\right)^{J}=\left(s^{J}\right)^{2}=\beta-\gamma r$ in $L$. Let $w=s s^{J}-s^{J} s=w^{J}$. Then $w \in S$, $s^{J} w+w s^{J}=2 w \circ s^{J}=s^{J} s s^{J}-\left(s^{J}\right)^{2} s+s\left(s^{J}\right)^{2}-s^{J} s s^{J}=0$ since $\left(s^{J}\right)^{2}$ is in $L \subset K$ and so commutes with $s$. Taking the $J$ of the previous expression we have sw+ws $=2 w \circ s=0$; adding the two we get $2 w \circ\left(s+s^{J}\right)=0$ for $w$ and $s+s^{J}$ in $S$. Therefore $w=0$ or $s+s^{J}=0$ since $S^{(+)}$is assumed to be a division algebra. In either case $s s^{J}=s^{J} s=\left(s s^{J}\right)^{J}$ is in $S,\left(s s^{J}\right)^{2}=s^{2}\left(s^{J}\right)^{2}=(\beta+\gamma r)(\beta-\gamma r)=\beta^{2}$ $-\gamma^{2} \alpha$ is in $F$. If $s s^{J}$ is not in $F$ then the application of Lemma 1, Part II contradicts our assumption that $S^{(+)}$is a division algebra. Therefore $s s^{J}=s^{J} S$ $=\delta \neq 0$ in $F, s^{J}=\delta s^{-1}$. Now $s+s^{J}$ is in $S,\left(s+s^{J}\right)^{2}=s^{2}+\left(s^{J}\right)^{2}+2 s s^{J}=2 \beta+2 \delta$ is in $F$ so that by a similar application of Lemma 1 we have $\eta=s+s^{J}$ in $F$, $\eta=s+\delta s^{-1}, s^{2}-\eta s+\delta=0$, which contradicts the primitiveness of $s$. This contradiction shows that our assumption that $S^{(+)}$is a division algebra was false, $S^{(+)}$is not a division algebra. q.e.d.

Now let $A$ be an associative central simple algebra of finite dimension over its center $F$ which is a crossed product over $F$ having an involution $J$ of the first kind. Then its degree $n=2^{m}$ and its maximal normal subfield is metacyclic, so that when $m>1, A$ certainly contains an imprimitive quartic field. Applying Theorems 1 and 2 to $A$ we have the following corollaries.

Corollary 1. Let $A$ be an associative central simple algebra of finite dimension over its center $F$ which is a crossed product having an involution of the first 
kind. Then either $S^{(+)}$is a field or $S^{(+)}$is not a division algebra.

COROLlary 2. Let $A$ be an associative central simple algebra of finite dimension over its center $F \supset R,[F: R]<\infty$. Let $A$ have an involution of the first kind. Then either $S^{(+)}$is a field or $S^{(+)}$is not a division algebra.

Corollary 2 follows immediately from Corollary 1 by the observation that the $A$ in Corollary 2 is cyclic.

This completes the discussion of the Jordan division algebras of Type 2. We now pass to the consideration of Jordan division algebras of Type 3. For the remainder of this paper, $J$ will denote an involution of the second kind, unless otherwise stated.

As remarked before, Theorem 1 of Part II enables us to limit our investigation to the algebras $S^{(+)}$, where $S$ is the set of all $J$-symmetric elements of a central associative division algebra $A$ of finite degree over its center $K$. It is well known that the set of all $J$-symmetric elements of $K$, that is, $S \cap K$, is a subfield $F$ of $K$ such that $K=F(\theta), \theta=-\theta^{J}, \theta^{2}=\alpha \neq 0$ in $F$. For the remainder of this paper, $A$ shall denote an associative central division algebra of finite degree $n$ over its center $K ; \theta, F$, and $S$ are defined as above, unless otherwise stated. Define $A^{S}$ to be the set of all elements of $A$ which commute with every element of $S$.

Lemma 3. $A^{s}=K$.

Let $x$ be in $A^{S}$ so that $y=y^{J}$ implies $x y=y x$. Let $z=-z^{J}$. Then $(\theta z)^{J}=\theta^{J} z^{J}$ $=(-\theta)(-z)=\theta z, \theta z$ is in $S ; x(\theta z)=(\theta z) x=\theta x z=\theta z x$ by the above italicized statement, $x z=z x$. Thus $z=-z^{J}$ implies $x z=z x$. Any $a$ in $A$ may be written in the form $a=y+z$, where $y=\left(a+a^{J}\right) / 2=y^{J}, z=\left(a-a^{J}\right) / 2=-z^{J}, x$ commutes with both $y$ and $z, x$ commutes with $a, x$ is in $A^{A}=K, A^{S} \subset K \subset A^{S}$, $A^{S}=K$. q.e.d.

Lemma 4. Let $x^{2}=\beta$ in $K, x$ in $S, x$ not in $K$. Then $S^{(+)}$is not a division algebra.

We first observe that since $x$ is not in $K, x$ is not in $A^{s}$ by Lemma 3. Therefore for some $z$ in $S, w=x z-z x \neq 0, w^{v}=z x-x z=-w$. Let $v=\theta w$. Then $v \neq 0$, $v^{J}=v$ so that $v$ is in $S, x \neq 0$ is in $S, 2(x \circ v)=x v+v x=\theta(x w+w x)=\theta\left(x^{2} z\right.$ $\left.-x z x+x z x-z x^{2}\right)=0$ since $x^{2}$ is in $K=A^{S}, x \circ v=0, S^{(+)}$is not a division algebra.

Lemma 5. Let $y^{2}=\beta$ in $K, y$ not in $K$. Then $S^{(+)}$is not a division algebra.

$y^{2}=\beta=a+b \theta$ where $a$ and $b$ are in $F$. Assume that $S^{(+)}$is a division algebra. $\left(y^{2}\right)^{J}=\left(y^{J}\right)^{2}=a-b \theta$ is in $K$. Let $u=y y^{J}-y^{J} y$, so that $u=u^{J}$ is in $S$. Then $u y+y u=y y^{J} y-y^{J} y^{2}+y^{2} y^{J}-y y^{J} y=0$ since $y^{2}$ is in $k$, (i) $u y^{J}+y^{J} u=0$ by applying $J$ to (ii) $u y+y u=0,2 u \circ\left(y+y^{J}\right)=u\left(y+y^{J}\right)+\left(y+y^{J}\right) u=0$ by adding (i) and (ii), for $u$ in $S$ and $y+y^{J}$ in $S$. The assumption that $S^{(+)}$is a 
division algebra then implies that either $u=0$ or $y+y^{J}=0$. In either case $y y^{J}=y^{J} y, y y^{J}$ is in $S,\left(y y^{J}\right)^{2}=y^{2}\left(y^{J}\right)^{2}=(a+b \theta)(a-b \theta)=a^{2}-b^{2}=c$ in $F \subset K$. If $y y^{J}$ is not in $K$ then $S^{(+)}$is not a division algebra by Lemma 4 . Therefore $y y^{J}$ is in $K, y y^{J}$ is in $S, y y^{J}$ is in $S \cap K=F, y y^{J}=e \neq 0$ in $F$. Also $y+y^{J}$ is in $S,\left(y+y^{J}\right)^{2}=y^{2}+\left(y^{J}\right)^{2}+2 y y^{J}=2 a+2 e$ in $F$. If $y+y^{J}$ is not in $K$ then $S^{(+)}$ is not a division algebra by Lemma 4. Therefore $y+y^{J}=f$ in $S \cap K=F$, $y+e y^{-1}=f, y^{2}-f y+e=0, f y=y^{2}+e$ in $K$ and $y$ would be in $K$ contrary to hypothesis unless $f=0, y=-y^{J}$. Let $v=\theta y$. Then $v^{J}=v \neq 0$ is in $S, v^{2}=\theta^{2} y^{2}$ is in $K, v$ is not in $K$. By application of Lemma 4 we get $S^{(+)}$not a division algebra. This contradicts our original assumption, so that $S^{(+)}$is not a division algebra.

THEOREM 3. Let $A$ be an associative central simple algebra over $K \supset R$, $[K: R]<\infty$. Let $n>1$ be the degree of $A$ over $K$ and let $J$ be an involution of the second kind over $K$ of $A$. Then $S^{(+)}$is a division algebra if and only if $A$ is a division algebra and $n$ is odd.

If $A$ is a division algebra and $n$ is odd, then $S^{(+)}$is a division algebra by the corollary to Theorem 2 of Part I. Conversely suppose $S^{(+)}$is a division algebra. Then $A$ is a division algebra by Theorem 1 of Part II. Suppose $n=2 m$ is even. Now $A$ is cyclic over $K, A=(Z, T, \gamma)$ over $K, Z$ is a cyclic field of degree $n$ over $K, A=Z+y Z+\cdots+y^{n-1} Z, y^{n}=\gamma$ in $K, z y=y z^{T}$ for $z \in Z$ where $T$ is the generating automorphism of the Galois group of $Z$ over $K$, and the indicated sum above is supplementary. Let $w=y^{m}$. Then $w$ is not in $K$, $w^{2}=y^{2 m}=y^{n}=\gamma$ in $K, S^{(+)}$is not a division algebra by Lemma 5 above. This contradiction shows that $n$ cannot be even. $n$ must be odd. q.e.d.

Our main result is that the only central Jordan division algebras $S^{(+)}$over an algebraic number field which are not fields are obtained by replacing ordinary multiplication by the quasi multiplication $a \circ b=(a b+b a) / 2$ in $S$, where $S$ is either

1. an associative central division algebra $D$ of odd degree over $F$, or

2 . the set of all $J$-symmetric elements of $D$ of odd degree over $F$, where $J$ is of the second kind.

The above result is an immediate consequence of Corollary 2 to Theorem 4 of Part I, Corollary 2 to Theorem 2 of Part II, and Theorem 3 of Part II.

\section{BIBLIOGRAPHY}

1. A. A. Albert, Non-associative algebras I, Ann. of Math. vol. 43 (1942) pp. 685-707.

2. - On Jordan algebras of linear transformations, Trans. Amer. Math. Soc. vol. 59 (1946) pp. 524-555.

3. - A structure theory for Jordan algebras, Ann. of Math. vol. 48 (1947) pp. 546-567.

4. N. Jacobson and F. D. Jacobson, Structure and representation of semi-simple Jordan algebras, Trans. Amer. Math. Soc. vol. 65 (1949) pp. 685-707.

University of Chicago, Chicago, Ill. 